Pak. J. Agri. Sci., Vol. 53(2), 393-398; 2016

ISSN (Print) 0552-9034, ISSN (Online) 2076-0906

DOI: $10.21162 / P A K J A S / 16.2240$

http://www.pakjas.com.pk

\title{
MOLECULAR IDENTIFICATION OF CHINESE Chroogomphus roseolus FROM PAKISTANI FORESTS, A MYCORRHIZAL FUNGUS, USING ITS-rDNA MARKER
}

\author{
Abdul Razaq1,*, Sobia Ilyas ${ }^{2}$ and Abdul NasirKhalid ${ }^{3}$ \\ ${ }^{1}$ Discipline of Botany, Faculty of Fisheries and Wildlife, University of Veterinary and Animal Sciences (UVAS), Ravi \\ Campus, Pattoki, Pakistan; ${ }^{2}$ Department of Botany, Lahore College for Women University, Lahore, Pakistan; \\ ${ }^{3}$ Department of Botany, University of the Punjab, Lahore 54590, Pakistan. \\ "Corresponding author's e-mail: ectomycorrhiza@gmail.com
}

Chroogomphus roseolus is a common mycorrhizal species exclusively found with Pinus wallichiana which has been collected from Himalayan moist forests of Pakistan. Taxonomically, this species is previously not described from Pakistan and also first time reported outside Chinese territory. In this study, Pakistani collection has been characterized on morphological and molecular basis. Macrosopic characters like size, shape and colour of pileus, stipe and spore are compared in Cinese European and North American literature for its identification. Its Internal Transcribed Spacers (ITS) of ribosomal DNA sequence was amplified using universal primer pair (ITS1f and ITS4) and nucleotide sequences queried in GenBank database using BLAST analysis where it matches $99 \%$ with sequences of the same species from China. Phylogenetic analysis, description, photographs and line drawings of Pakistani collection confirmed as Chroogomphus roseolus. . The exclusive presence of this species Pinus wallichiana indicates that it can be used in mycorrhization control in afforestation and reforestation of Pinus wallichiana vegetation.

Keywords: Gilled bolete, Himalayan forests, mycoforestry, Pinus wallichaiana, ribosomal DNA, macro-fungi, mycorrhiza, molecular marker.

\section{INTRODUCTION}

Chroogomphus (Singer) O.K. Mill. is well distributed in Chinese territory and seven out of total twenty species of this genus were found in China (Li et al., 2009). This genus is mainly characterized by pale orange to ochraceous lamellae when young, an ochraceous pileal trama, a moist to viscid pileus covering and a mycelium at the base of the stipe consisting of amyloid hyphae (Miller, 1964; Miller and Aime, 2001; Li et al., 2009). This genus is economically very important and its basidiomata are used as food and medicinal herbs (Xie et al., 1986; Yu and Liu, 2005; Dai and Tolgor, 2007).

Based on previous records, Chroogomphus species are not well documented and reported from Pakistan and only two species, Chroogomphus helveticus (Singer) M.M. Moser and C. rutilus (Schaeff.) O. K. Mill., have been reported from Pakistani forests but their detail descriptions and herbarium specimens were not available (Ahmad et al., 1997). The scarcity of reports on this genus is not due to lack of sufficient material but this may be due to limited understanding and poor documentation. Pakistani forests are famous for their biodiversity richness of plants, animals and macrofungi. More than 300 species of gilled fungi have been listed from only Himalayan wet temperate forests belonging to different ecological groups like mycorrhizal, saprophytic, and wood decaying (Ahmad et al., 1997; Razaq et al., 2012a, 2013a,b); Ilyas et al., 2013a,b). All these fungi are playing very important role in the sustainability and dynamics of these forests though wet forests of Pakistan are under a severe pressure of unsustainable practices (Irshad and Khan, 2012). Pine vegetation, in Himalayan moist temperate areas, is under huge anthropogenic pressure for its consumption as fuel wood (35Kg/day/household), timer (7trees/season/household) and also these forests are under pressure for fodder and grazing requirements (Jamal and Khadija, 2009). Urbanization and anthropogenic activities in these naturally growing forests are depleting the forest cover area drastically although the forestry department is working to cope with these unsustainable practices by increasing the number of annual seedlings. The survival rate of these seedlings is limited by subsequent long winter snowfall and dryness of soil, and nearly 25\% succeed to root under stress conditions (Marx and Cordell, 1989). This survival rate and growth of plants can be multiplied by using mycoforestry or mycorrhization control approach which means the inoculation of compatible mycorrhizal fungus with fresh seedlings and the practical demonstration and economic impact of this practice are evident in United States of America and France (Castellano and Molina, 1989; Marx and Cordell, 1989; Selosse et al., 2000). However, in order for mycorrhizal inoculation to become a routine practice in nurseries, it is necessary to 
develop methods for identification and screening of compatible fungus.

In this study, a new record of mycorrhizal Chinese Chroogomphus species, C. roseolus, is being described from Pakistan using morpho-anatomical details and molecular data. This is first record of this species outside the Chinese territory as this genus has narrow distribution range (Li et al., 2009). Chroogomphus roseolus is a gilled bolete with carmine-rose coloured basidiomata and non-amyloid hyphae on its stipe base. This species is always found in association with Pinus wallichina or with pine species of sub-genus Pinus. The hyphal network of this species beneath pine tree soil plays a key role in the growth of tree by assisting the uptake of water, soil nutrients, nitrogen and phosphorus, and providing resistance against root pathogens, heavy metals and harsh environment (Smith and Read, 1997; Halling, 2001; Ilyas et al., 2013b). Morpho-anatomical and molecular description which is based on internal transcribed spacer of ribosomal DNA (ITS-rDNA) will be useful for its re-collection and reliable identification which ultimately will lead to its culture raising and inoculation with pine seedlings for afforestation and reforestation for sustainable ecosystem.

\section{MATERIALS AND METHODS}

Morphological characterization: Basidiocarps of this species were collected from wild forests of Khanspur, Himalyan moist temperate forests of Pakistan at 2400-2580 m a. s. 1. and its fruiting bodies dugout carefully with the help of a sharp knife and photographed on the spot. Morpho-anatomically the specimen was described following $\mathrm{Li}$ et al. (2009). Morphological characters were noted in the field. Sections of lamellae were prepared stained with Congo Red and Melzer's reagent. Size dimensions were determined for 25 basidiospores, 20 basidia, 20 cystidia and 20 pileal element from each basidioma under the light microscope equipped with camera lucida.

Molecular characterization and phylogenetic analysis: Dried gill tissue (approx. 100mg) of basidiocarps was taken for DNA analysis and material macerated in liquid nitrogen using pestle mortar. The macerated tissue was gently shifted in to $1.5 \mathrm{ml}$ centrifuge tube and the genomic DNA was extracted following protocol given by Razaq et al. (2013b). The target region, Internal Transcribed Spacers (ITS) part of rDNA was amplified using fungal specific ITS1F and universal ITS4 primers (White et al., 1990). Polymerase Chain Reaction (PCR) was performed in $20-\mu 1$ reaction volume following the profile given by Gardes and Bruns (1993). The size of PCR product was determined on $1.5 \%$ agarose gel in gel documentation system (UVtec, Avebury House, Cambridge CB4 1QB UK) using default setting. PCR product was directly sequenced in both directions using the same pair of amplification primers (Macrogen, Korea).
The generated sequence was edited manually using BioEdit sequence alignment editor version 7.0.9.0 (Tom Hall, Ibis Biosciences, Carlsbad, California). For initial comparison and alignment of the sequence, BLAST (Basic Local Alignment Search Tool) analysis was performed using the National Center for Biotechnology Information (NCBI), USA database. For further phylogenetic analysis and alignment of sequence, closely related sequences were retrieved from GenBank. The newly generated sequence and those which retrieved from the GneBank are aligned by using Cluatal W program of Molecular Evolutionary Genetics Analysis (MEGA) software (Tamura et al., 2011). Maximum likelihood (ML) analysis was performed in the MEGA.5 software using Jukes-Cantor model of nrITS sequences and Nearest-Neighbor-Interchange (NNI) as ML heuristic search method to determine the relationship between Pakistani and Chinese collections. . Consensus nucleotide sequence of $C$. roseolus was submitted to European Molecular Biology Laboratory (EMBL) database.

\section{RESULTS}

Morphology and taxonomy:

Chroogomphus roseolus: Yan $\mathrm{C} . \mathrm{Li} \& \mathrm{Zhu} \mathrm{L}$. Yang, fungal Divers. 38, 2009. Fig.1, A-E

Pileus $3.0-6.5 \mathrm{~cm}$ wide, plano-covex to plane, pinkish brown, uniform over whole surface, smooth to fibrillose, soft, shiny; margins dentate, flexuous, fragile; context moderately thick, firm, unchanging when bruised or cut. Lamellae concolorus to pileus, broad, arched, decurrent, subdistant, fleshy, brittle, edges entire; lamellulae two different lengths, one marginal other end in the middle of the cap. Stipe $1.5-2 \times 0.3-0.42 \mathrm{~cm}$, concolorous with pileus, smooth to fibrilose, striated, cylindrical with mid part tapered; context orange, without volval scales or zones and ring. Odor and taste not recorded. Basidiospores 15.0-19.5 $\times 6.5-8.8 \mu \mathrm{m}$, avl $\times$ avw $=17.5-7.8$ $\mu \mathrm{m}, \mathrm{Q}=2.23-2.3$, avQ=2.25; apiculate, thin walled, ellipsoid to oblong, hyaline to pale yellow in $5 \% \mathrm{KOH}$, inamyloid, yellow in Melzer's reagent, smooth, entire, distal and proximal ends broader less than middle. Basidia 35.5-43.5 $\times$ 9.5-13.0 $\mu \mathrm{m}, 4$-spored, sharp ended sterigmata, hyaline to pale yellow in $5 \% \mathrm{KOH}$, thin walled, sub-clavate to clavate with oil like contents. Hymenium trama hyphal, regular, parallel. Pleurocystidia $83.0-110.5 \times 14.0-17.5 \mu \mathrm{m}$, thin walled, hyaline, broadly clavate, olive green grunnules concentrated on different spots, septate base. Cheilocystidia are similar to Pleurocystidia. Pileus covering a cutis with erect or ascending, interwoven, hyaline, branched hyphae, terminal elements, 77.0-108.5 × 6.0-11.5 $\mu \mathrm{m}$, light brown pigmentation.

Habitat and distribution: Pakistan: Khyber Pakhtankhaw, Abbotabad, Ayubia-Khanspur (Halley pad side), at 2400$2580 \mathrm{~m}$ a. s. 1., on the ground humified soil under Pinus 

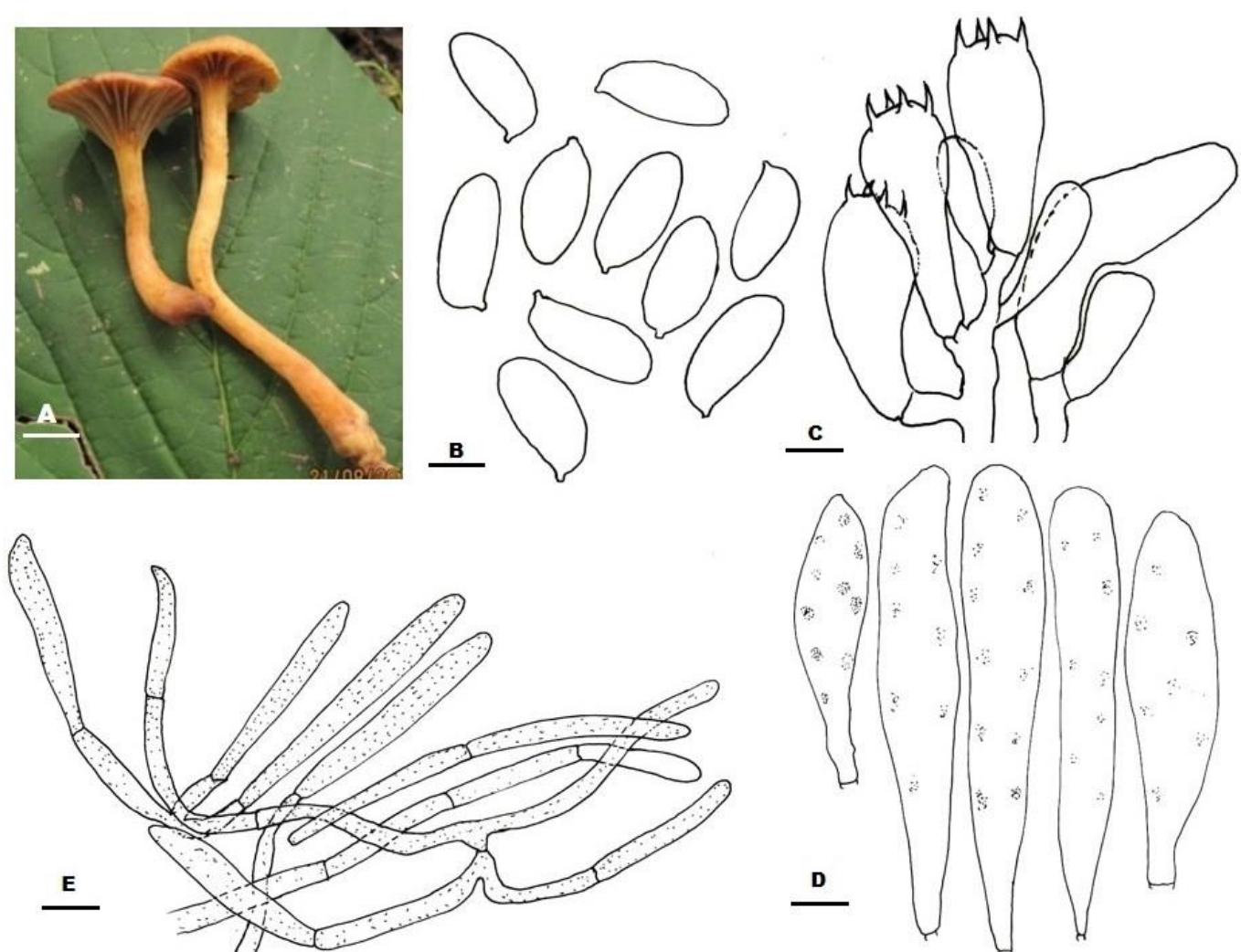

Figures 1A-E. Chroogomphus roseolus A. Basidiomata with pileus and lamellae. B. Basidiospores. C. Hymenium with basidia. D. Cystidia E. Pileal covering elements. Bars $=A=1.2 \mathrm{~cm}, \mathrm{~B}=3 \mu \mathrm{m}, \mathrm{C}=7 \mu \mathrm{m}, \mathrm{D}, \mathrm{E}=13.5 \mu \mathrm{m}$,

wallichina vegetation, solitary, Abdul Razaq, KP-56, 23-082010; submitted to herbarium, Department of Botany, University of the Punjab, Lahore. LAH. 13081056.

rDNA sequence and phylogenetic analysis: The target region copmrising of internal transcibed spacers (ITS1 \& ITS2) and $5.8 \mathrm{~S}$ of rDNA gave fragments of approximately $700 \mathrm{bp}$ on amplification in polymerase chain reaction (PCR) using fungal universal primers pairs (ITS1f \& ITS4). Initial BLAST analysis of nucleotide sequences revealed that Pakistani collection matches maximum with Chroogomphus roseolus (GenBank accession \# EU706329.1) 99\%, Query coverage 100, E-value 0 submitted from China. All the sequences in Blast analysis of Pakistani sequence belong to the same genus, Chroogomphus, or different genera of the same family. Other closely related sequences in the Blast below the closest sequence showed $98 \%, 98 \%, 94 \%$ and $93 \%$ with C. helveticus (GenBank accession AF205642.1, USA), C. helveticus (GenBank accession \# AF205650.1, Germany), C. rutilus (GenBank accession \# GQ367894.1, Canada) and $C$. filiformis (GenBank accession \# FJ513327.1, China) respectively. Phylogenetic analysis is conducted to determine the clustering of this local species with other species from different regions of the world.
A total 36 sequences of Chroogophus species were included in phylogenetic analysis possibly those which are published and have some close relationships to sequence included from Pakistan. Only one species belonging to this genus was collected and sequenced from Pakistan and its sequence was analyzed in this phylogenetic tree (Fig. 2). The sequences included in phylogenetic analysis had 1195 characters, from which 703 characters were used in final analysis after trimming the alignment from both 5' and 3'ends. In phylogenetic analysis gaps were treated as data. Almost all sequences included separate into two major clade which were labeled as sections. The species which share common conserve region on their ITS-rDNA are clustered together in Chroogomphus clade. Generally the species of sect. Chroogomphus clade are: Chroogomphus rutilus (HM049561.1, HM049562.1, HM049563.1, HM049564.1, EU791582.1, EU791580.1), C. orientirutilus (EU706326.1, EU706327.1, EU706328.1, EU706325.1), C. purpurascens (FJ481128.1， EU706330.1， EU706331.1， EU706332.1, EU706333.1, FJ652072.1), C. filiformis (EU706323.1, EU706324.1, FJ513326.1, FJ513327.1) and C. confusus (EF423623.1, EF423622.1, EF423621.1). The other major clade recoved in phylogenetic tree is labelled as sect. Floccigomphus (S. Imai) O.K. Mill. This clade contains $C$. 


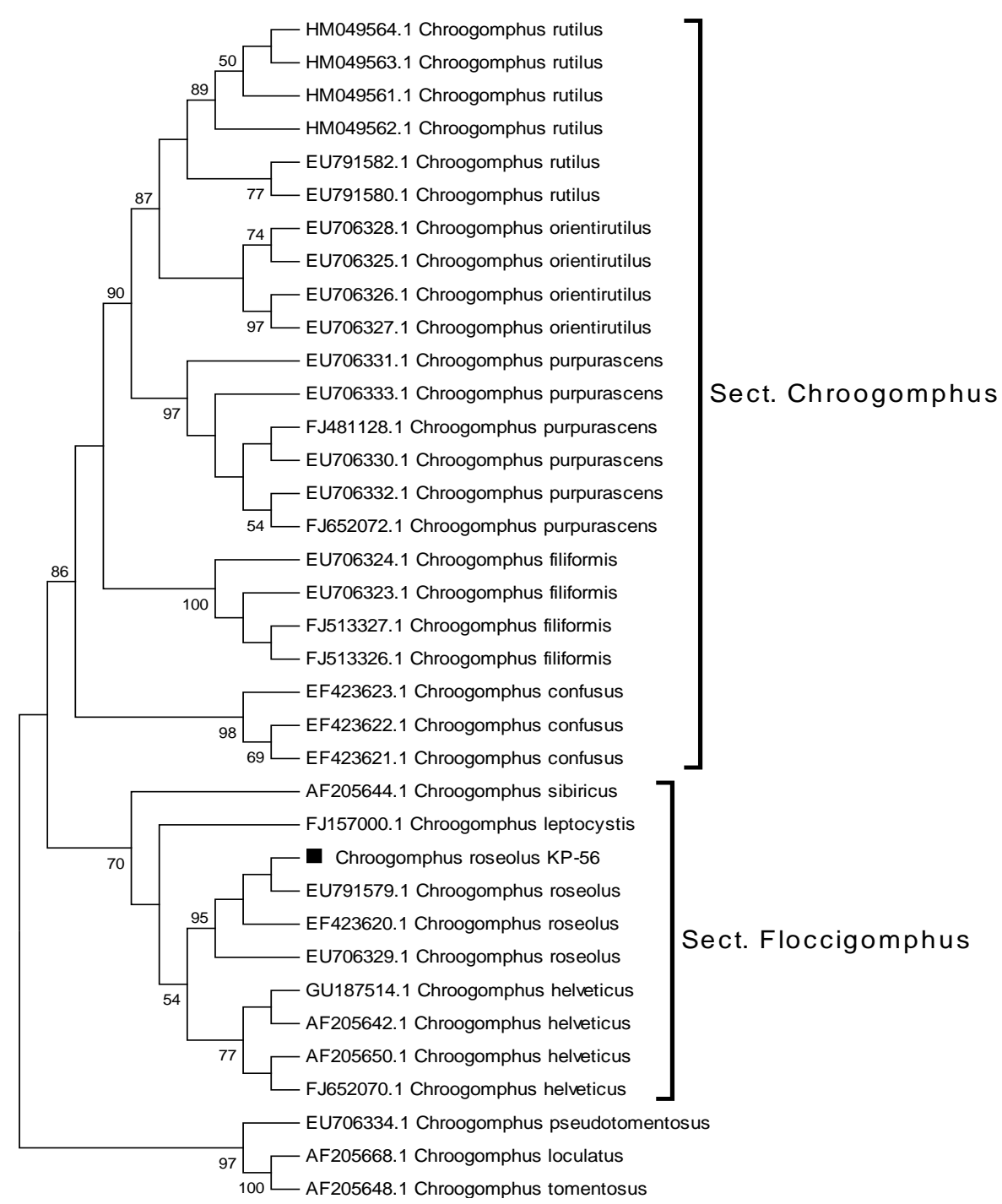

Figure 2. Phylogenetic analysis of Chroogomphus roseolus collected from Pakistan based on nrITS-rDNA regions. This tree is based on maximum likelihood method using Jukes-Cantor model. The values given above branches represent bootstraps. The species collected from Pakistan have been labeled (ם).

leptocystis (Singer) O. K. Mill. (AF205644.1), C. helveticus (GU187514.1, AF205642.1, AF205650.1, FJ652070.1) and C. sibericus (Singer) O.K. Mill. and C. roseolus (EU706329.1, EF423620.1, EU791579.1) are all together in a clade with high bootstrap support (Fig. 2). Pakistani species lies among the members of sect. Floccigomphusclade. $C$. helveticus and $C$. roseolus form sister clade to each other while the Pakistani collection very clearly clustered with isolates of $C$. roseolus. No doubt the bootstrapping is not well supported yet the reshuffling of the sequence may remain inside the same clade.

\section{DISCUSSION}

Chroogomphus roseolus is characterized by pink to purplish pink pileus with conclorous lamellae which are sub-decurrent in attachment, pinkish mycellium at the base of stipe, boletoid basidiospores, and its specific mycorrhizal association with pine vegetation for uptake of water and nutrients ( $\mathrm{Li}$ et al., 2009). Pakistani collection is commonly found in association with Pinus wallichiana which is abundantly covering the Galyat areas of Himalayan moist temperate forests of Pakistan. Pakistani collection has very close resemblance with Chinese collection described by Li et al. (2009). The coloration of pileus, stipe and gills, and the mycelium on stipe base are same as described by the authors of this species. 
Pakistani collection has some more brownish tans and found associated with Pinus wallichiana of sub-genus strobus make our collection very close to $C$. helveticus but brownish pileal squamules, larger basidiospore size $(17-23 \times 6.8-8 \mu \mathrm{m})$ of $C$. helveticus and phylogenetic analysis place our collection with C. roseolus.

In the phylogenetic analysis, Chroogomphus species retrieved from GenBank clustered into two sections: sect. Chroogomphus and sect. Floccigomphus, and Pakistani collection lies on latter section members with $C$. roseolus, $C$. leptocystis and $C$. helveticus. Pakistani collection very clearly clustered in sub-clade of Chines sequences of $C$. roseolus (Fig. 2). Our molecular results are in accordance with Li et al. (2009) and Miller, (2003) also placed two closely related species , C. helveticus and C. leptocystis, together in the same clade. Molecular results confirms the occurrence of $C$. roseolus in Pakistani forests but with blue pine, a different host.

Previously, Ahmad et al. (1997) reported C. helveticus from the same area of $C$. roseolus species with blue pine but the lack of herbarium specimen, description and illustration in literature lead us to conclude that Pakistani forests have only C. roseolus associated with blue pine. Morphologically, the both $C$. helveticus from the same area of $C$. roseolus are very closely related but the molecular analysis is good tool to differentiate these two species (Li et al., 2009). Secondly, Chroogomphus species clustering in clade I (C. helveticus, $C$. leptocystis \& $C$. sibericus) of Miller, (2003) and clade II ( $C$. helveticus, C. leptocystis, C. sibericus \& C. roseolus) of Li et al., (2009) are closely related species which belong to different geographical regions. All these species can have mycorrhizal host-switch from one to another (Li et al., 2009). In the same way, at species level, there is a strong possibility for $C$. roseolus to share two different pine species or may be the time of evolution of Pinaceae and Chroogumpus was same for colonization. There are also high chances of $C$. helveticus, collection from Pakistan previously, being $C$. roseolus because the former is native to Europe while latter is endemic to Asian territory. C. roseolus is well distributed on both sides of Himalaya, Pakistani and Chinese forming specific mycorrhizal association with pine vegetation.

Conclusion: Chroogumpus roseolus is first time recorded from Pakistan after China and it is purely Asian species and always found associated mutulistically with pine vegetation on both sides, China and Pakistan. Previously, reported $C$. helveticus with Pinus wallichiana from Galyat region of Himalayan moist temperate forests of Pakistan is actually seems $C$. roseolus on their close morphological similarities which could not be reaffirmed as the herbarium specimen of C. helveticus which is a European native species, is not available. Chroogumpus roseolus might has great potential for mycoforestry or mycorrhization control for plantation of
Pinus wallichiana as this fungus is compatible for pine vegetation. The large scale inoculation culture of $C$. roseolus is necessary for reforestation and reforestation practices.

Acknowledgement: Authors are sincerely thankful to Miss Nousheen Yousaf, Department of Botany, University of the Punjab, Lahore for suggesting good comments to finalize the manuscript.

\section{REFERENCES}

Ahmad, S., S.H. Iqbal and A.N. Khalid. 1997. Fungi of Pakistan. Sultan Ahmad Mycological Society of Pakistan, Department of Botany, University of the Punjab, Quaid-e-Azam campus, Lahore, PAKISTAN.

Castellano, M.A. and R. Molina. 1989. Mycorrhizae, pp.101167. In: T.D. Landis, R.W. Tinus, S.E. McDonald and J.P. Barnett (eds.), The Biological Components: Nursery Pests and Mycorrhizae- The Container Tree Nursery Manual. US Department of Agriculture, Forest Service, Washington, DC, USA.

Dai, Y.C. and B. Tolgor. 2007. Illustration of Edible and Medicinal Fungi in Northeastern China (in Chinese). Science Press, Beijing, China.

Gardes, M. and T.D. Bruns. 1993. ITS primers with enhanced specification of mycorrhizae and rusts. Mol. Ecol. 2:113118.

Halling, R.E. 2001. Ectomycorrhizae: Co-Evolution, Significance, and Biogeography. Ann. Missouri Bot. Gard. 88:5-13.

Ilyas, S., A. Razaq and A.N. Khalid. 2013a. Inocybe nitidiuscula and its ectomycorrhiza associated with Alnus nitida from Galyat, Pakistan. Mycotaxon.124:247-254.

Ilyas, S., A. Razaq and A.N. Khalid. 2013b. Molecular investigations to determine the ectomycorrhizal habit of Lactarius sanguifluus associated with coniferous and deciduous vegetation of Galyat, Khyber Pakhtunkhwa, Pakistan. Int. J. Agric. Biol. 15:857?863.

Irshad, S. and S. Khan. 2012. Impacts of protection on floral diversity of Himalayan moist temperate forests of Galyat, Pakistan. J. Environ. 1:119-125.

Jamal, S. and Khadija. 2009. Watershed issues assessment in Galyat. M.Sc. Diss., Hazara Univ., KPK, Pakistan.

Li, Y.C., Z.L. Yang and B. Tolgor. 2009. Phylogenetic and biogeographic relationships of Chroogomphus species as inferred from molecular and morphological data. Fungal Divers. 38:85-104.

Marx, D.H. and C. E. Cordell. 1989. The use of specific ectomycorrhizas to improve artificial forestation practices, pp.1-25. In: J.M. Whipps and R.D. Lumsden (eds.), Biotechnology of Fungi Improving Plant Growth. New York, USA. 
Miller, O.K. 1964. Monograph of Chroogomphus (Gomphidiaceae). Mycologia 56:526-549.

Miller, O.K. 2003. The Gomphidiaceae revisited: a worldwide perspective. Mycologia 95:176-183.

Miller, O.K. and M.C. Aime. 2001. Systematics, ecology and world distribution in the genus Chroogomphus (Gomphidiaceae), pp.314-333. In: J.K. Misra and B.W. Horn (eds.), Trichomycetes and other fungal groups. Enfield New Hampshire: Science Publishers, Inc.

Razaq, A., A.N. Khalid and S. Ilyas. 2012a. Tricholomopsis flammula Métrod ex Holec (Basidiomycota, Agaricales)an addition to the mushroom flora of Pakistan based on molecular identification. Pak. J. Bot. 44:413-416.

Razaq, A., A.N. Khalid and E.C. Vellinga. 2012b. Lepiota himalayensis sp. nov. (Basidiomycota, Agaricales), a new species from Pakistan. Mycotaxon.121:319?325.

Razaq, A., A.N. Khalid and S. Ilyas. 2013a. Molecular identification of Lepiota acutesquamosa and L. cristata (Basidiomycota, Agaricales) based on ITS-rDNA barcoding from Himalayan moist temperate forests of Pakistan. Int. J. Agric. Biol.15:3130318.

Razaq, A., A.N. Khalid and S. Ilyas. 2013b. Molecular identification of Marasmius oreades - an edible mushroom from Pakistan based on ITSrDNA data. Pak. J. Agri. Sci. 50:415-419.
Selosse, M.A., D. Bouchard, F. Martin and F. Le Tacon. 2000. Effect of Laccaria bicolor strains inoculated on Douglasfir (Pseudotsuga menziesii) several years after nursery inoculation. Can. J. Forest Res. 30:360-371.

Smith, S.E. and D.J. Read. 1997. Mycorrhizal Symbiosis, $2^{\text {nd }}$ Ed. Academic Press, San Diego, USA.

Tamura, K., D. Peterson, N. Peterson, G. Stecher, M. Nei and S. Kumar. 2011. MEGA5: Molecular evolutionary genetics analysis using maximum likelihood, evolutionary distance, and maximum parsimony methods. Molecular Biol. Evol. 28:2731-2739.

White, T.J., T. Burns, S. Lee and J. Taylor. 1990. Amplification and direct sequencing of fungal ribosomal RNA genes for phylogenetics, pp.315-322. In: M.A. Innis, D.H. Gelfand, J.J. Sninsky and T.J. White (eds.), PCR Protocols: A guide to methods and applications. San Diego: Academic Press, USA.

Xie, Z.X., Y. Wang and B. Wang. 1986. Illustration of Agarics of Changbai Mountains, China (in Chinese). Changchun: Jilin Scientific and Technology Press, China.

Yu, F.Q. and P.G. Liu. 2005. Species diversity of wild edible mushrooms from Pinus yunnanensis forests and conservation strategies (in Chinese). Biodiversity Sci.13:58-69. 